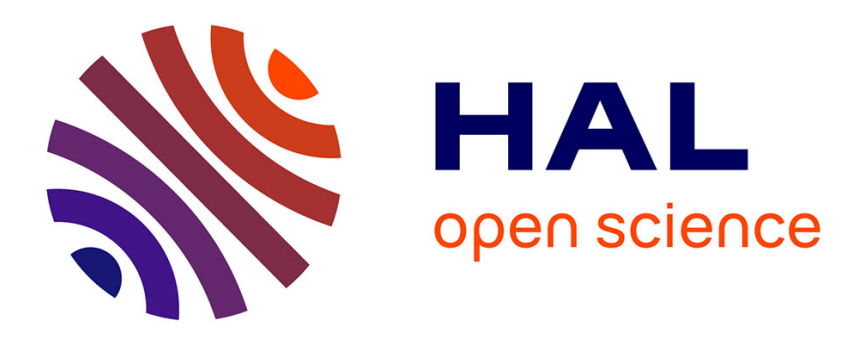

\title{
Les Ambivalences du devenir d'un écomusée : entre repli identitaire et dépossession \\ Serge Chaumier
}

\section{To cite this version:}

Serge Chaumier. Les Ambivalences du devenir d'un écomusée : entre repli identitaire et dépossession.

Publics et musées, 2000, n¹7-18, pp. 83-113. hal-00454026

\author{
HAL Id: hal-00454026 \\ https://hal.science/hal-00454026
}

Submitted on 13 Feb 2010

HAL is a multi-disciplinary open access archive for the deposit and dissemination of scientific research documents, whether they are published or not. The documents may come from teaching and research institutions in France or abroad, or from public or private research centers.
L'archive ouverte pluridisciplinaire HAL, est destinée au dépôt et à la diffusion de documents scientifiques de niveau recherche, publiés ou non, émanant des établissements d'enseignement et de recherche français ou étrangers, des laboratoires publics ou privés. 


\section{PUB L I C S et M U SÉ E S}

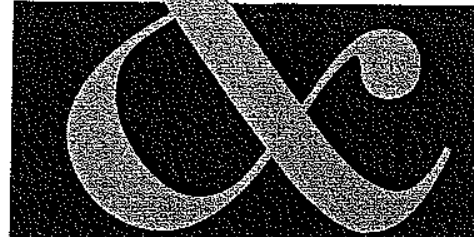

L'ÉCOMUSÉE :

RÊVE OU

RÉALITÉ

FRANCOIS MAIRESSE



Nina Gorgus

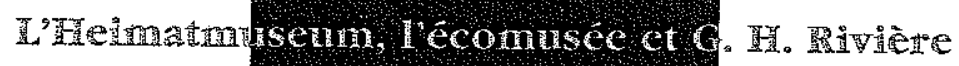

OCTAVE DEBARY

L'éconusec est mort, vive le malsée

Serge Chaumier

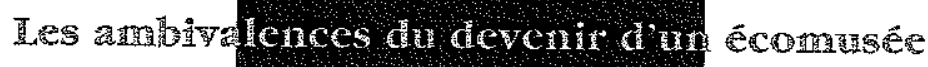

SASKIA COUSIN

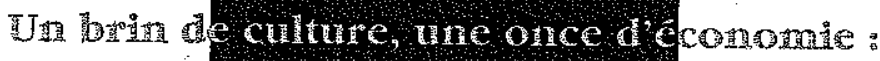

écomusée et conomuste

ALEXANDRE Delarge

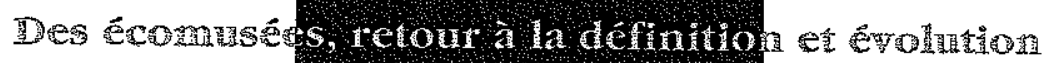

RAYMOND

\section{De LA Rocha-Miule}

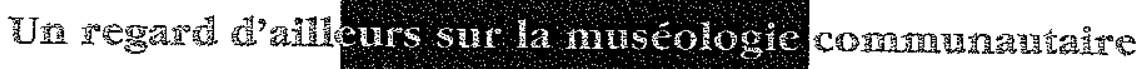

$D O O U 11, N \mathrm{~s}$

THMOGNGES CT ENTRETLENS 


\title{
LES AMBIVALENCES DU DEVENIR D'UN ÉCOMUSÉE : ENTRE REPLI IDENTITAIRE ET DÉPOSSESSION
}

\author{
$\mathrm{L}$ \\ Le propos de cet article \\ pourra paraître à certains \\ polémique. Il s'efforce de \\ débusquer à partir d'un cas \\ particulier des logiques \\ couramment convoquées \\ dans le champ des musées \\ d'identité. Il nous a paru \\ heuristique de relire les \\ théories fondatrices de \\ l'écomuséologie, leurs inter- \\ prétations depuis trente ans \\ et de les confronter avec \\ l'analyse singulière d'un \\ site, particulièrement aux \\ positions des acteurs inter- \\ viewés dans le cadre d'une \\ enquête de terrain.
}

Nous proposons de réfléchir ainsi aux écarts et aux évolutions, si ce n'est aux glissements de sens qui ont eu lieu. Nous montrerons en quoi la professionnalisation et l'institutionnalisation des structures contraignent à repenser, redéfinir ou envisager l'abandon du concept d'écomusée. La rencontre entre bénévoles fondateurs et professionnels occasionne fréquemment des incompréhensions, des frictions, des heurts. Cette récurrence conflictuelle, loin d'être fortuite, accidentelle ou conjoncturelle, révèle des contradictions plus profondes entre théories, histoire de la muséologie, histoire des sites et logiques économiques et professionnelles présentement à l'œuvre. Il conviendrait certainement d'élargir et de préciser le propos en amenant d'autres exemples, en détaillant certains aspects, en articulant la thèse aux points de vue d'autres acteurs. Le lecteur comprendra que le pari était impossible dans le cadre d'un article ${ }^{38}$. Nous avons donc choisi de cibler la démonstration sur un site et de privilégier le discours des bénévoles et des locaux, compte tenu que celui des professionnels est mieux connu et s'exprime déjà largement au travers de la théorie. Notre démarche, en tant que sociologue, a été de nous tenir à égale distance des deux parties, prenant le risque évident d'irriter l'une et l'autre. Il ne s'agit pas, en l'occurrence, de mettre en cause le travail de qualité, la rigueur et l'excellence des professionnels, pas plus que de ridiculiser un discours localiste identitaire, mais de pointer des logiques sociales contradictoires qui s'expriment au travers des 
divergences et des conflits. Les uns sont dans une logique disciplinaire quand les autres répondent à une logique du territoire. Il s'agit moins de savoir qui est dans le vrai que de comprendre le fondement qui articule les actions des uns et des autres. L'exemple condense les différends relatifs à l'implication et au respect des volontés locales, aux effets de la professionnalisation, à la destination et aux fonctions sociales du lieu, aux discours et aux moyens muséographiques utilisés, et, par conséquent, aux médiatisations retenues. Loin de représenter un cas unique ou anecdotique, l'exemple retenu est symptomatique de problématiques récurrentes. Il nous semble exprimer au mieux ce qui s'opère de façon plus ou moins affirmée ailleurs. Simplement, les métamorphoses actuelles du site, ses hésitations à se professionnaliser, les réticences et les vigilances de ses fondateurs en font un terrain de recherche particulièrement pertinent. Nous tairons le nom du lieu et des acteurs car leur désignation a peu d'importance. Ce sont les mécanismes qui nous importent.

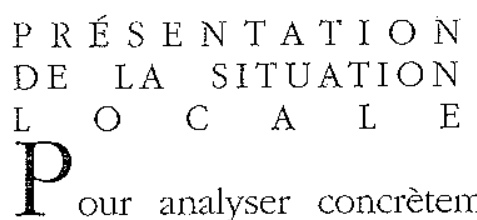

$\mathrm{P}$ enjeux, il nous faut présenter succinctement les parties en présence. Petite commune de quelque 3000 habitants, la commune est assez représentative des micro-sociétés traditionnelles. L'activité économique a longtemps reposé sur la production d'un produit de consommation particulier. Cette spécificité marque profondément le territoire, l'économie et la culture de ses habitants. Dans la fièvre muséale des ànnées quatre-vingt, et avec le soutien de Georges Henri Rivière et d'André Desvallées, une volonté se manifeste pour mettre en valeur le patrimoine local. S'appuyant sur l'initiative de passionnés, la municipalité acquiert un domaine d'un ancien producteur et le met à disposition d'une association culturelle. Le site est un endroit incontournable dans la topographie et la mémoire locale. L'association se charge de remplir la maison de maître avec pour mission de "montrer comment c'était avant". Devenu écomusée en 1982, la visite guidée du site est principalement consacrée aux collections liées aux modes de vie des classes dirigeantes locales du tournant du siècle dernier. S'y ajoute une maison des "manouvriers" présentant très succinctement la vie des ouvriers agricoles, et deux bâtiments consacrés à la production. Contrairement à ce que l'on supposerait, l'accent n'est pas mis principalement sur cette activité, pourtant singulière. La personnalité du fondateur impose le respect et continue à faire autorité. Mais très vite d'autres vocations émergent et une nouvelle association, dédiée spécifiquement au lieu, fédère dans cette petite commune un nombre impressionnant d'habitants. Si 50 personnes sont adhérentes et actives dans l'association, c'est un cercle beaucoup plus large qui se trouve partie prenante des animations. Si bien que le lieu se met à compter dans les orientations de politique locale. Ce d'autant plus que l'éco- musée locale donc 1 ressoul image

UN LEE

A

fédère tion ti se rer prend de no massi avant récolt "évén week Au $\mathrm{m}$ ? attire local Écom S'affir accor viales sance le po des $c$ ment: s'opf à ani cipal: sée,

conft dont se trí à sav très

conv

sous

ense:

inter ristiq phie reval semt 
naire as de ticule rela- pro, aux uent, anecécurus ou I site, as de inent. 1 peu

st les ience. eprélongurticulet la vingt, , une local. it un iation : et la naitre solnu$\mathrm{x}$ coltourrriers" - bâtiposeit $\sin -$ i faire assomune rentes zui se compl'éco- musée présente une activité qui continue à être le symbole de l'économie locale et qui n'est pas sans connaître des difficultés. Les enjeux ne sont donc pas que culturels, la valorisation d'un savoir faire local, d'un lieu de ressource pour le développement économique et la mise en avant d'une image positive de la ville sont recherchées.

\section{UN LIEU DE VIE ET UN ENJEUT DE POUVOIR}

Au-delà des tensions et des divisions parfois exacerbées, le site fédère des énergies et des volontés. Il semble correspondre à la définition traditionnelle de l'écomusée qui affirme les liens sociaux en faisant se rencontrer des acteurs locaux désireux d'exprimer leur identité et de prendre en main leur destin commun. Ainsi l'association organise-t-elle de nombreuses animations et des rendez-vous annuels où se déplace massivement la population. Des fêtes sont organisées où chacun met en avant ses talents ou se forme à de nouvelles missions. À la journée des récoltes potagères succède la fête du pain, la cueillette des fruits, des "événementiels" avec de petites expositions présentées le temps d'un week-end ou encore des démonstrations de vannerie ou de broderie. Au moins une fois par mois durant la saison d'ouverture, l'écomusée attire un public fidèle et curieux. Ces petites manifestations à caractère local (fête du miel, récolte des plantes aromatiques, élection de Miss Écomusée, fêtes des miches décorées...) permettent à l'association de s'affirmer et de développer de nouvelles activités. Une véritable liesse accompagne les festivités avec des ambiances très animées et conviviales. La socialité qui se développe durant ces moments de réjouissances collectives génère des dons et accroît l'audience, la crédibilité et le poids politique de l'association. Car l'association regroupe une partie des opposants à l'équipe municipale et se trouve donc en partie instrumentalisée. Le musée est investi par une partie de la population, mais s'oppose à d'autres qui y sont hostiles. L'écomusée contribue de ce fait à animer la vie politique locale, et les implications citoyennes. La municipalité, qui a plusieurs fois vacillé sur les questions relatives à l'écomusée, se montre extrêmement prudente et modérée. Ainsi un premier conflit est repérable entre la municipalité et l'opposition municipale dont l'écomusée est le fer de lance. Toutefois la divergence disparaît et se transforme en union sacrée dès qu'apparaît le troisième acteur du site, à savoir l'équipe de professionnels des musées. En effet, la situation est très particulière et doit être rapidement expliquée. À la suite d'une convention signée à la demande de la municipalité, l'écomusée est placé sous l'autorité scientifique d'un conservateur qui gère par ailleurs un ensemble de sites dispersés sur un large territoire. Les professionnels interviennent dans le domaine de la promotion et du développement touristique, de la production audiovisuelle, de la recherche, de la muséographie, de la gestion des collections et de l'action pédagogique. En revanche, ils ne s'occupent pas du fonctionnement général. Le schéma semble donc être idéal et correspond aux missions définies par l'écomu- 
séologie de professionnels à l'écoute et au service d'une expression locale.

Si lassociation dés amis de l'écomusée se montre "gourmande" quand ił s'agit de bénéficier de la force de frappe, des moyens et des compétences des professionnels en matière de promotion, notamment au travers des effets de réseau, en revanche la situation se complique dès lors que des actions sont entreprises à l'intérieur même de l'espace muséal. L'équipe des professionnels ne se trouvant impliquée ni dans la gestion quotidienne du site, domaine réservé de l'association, ni dans la vie locale, puisque ne résidant pas sur place, les interventions sont ponctuelles et vécues par les acteurs locaux comme des impositions venues de l'extérieur. Si tous s'accordent sur la nécessité d'une rénovation du site face aux graves difficultés actuelles - les collections sont installées dans des bâtiments non conformes aux normes de conservation en vigueur et aux nouvelles exigences d'accueil et de sécurité du public -, les avis diffèrent sur les moyens à mettre en œuvre. Les conflits éclatent dès lots qu'il s'agit de savoir quel aménagement ou quelle rénovation il convient de réaliser, par quí, à quel coût, comment, selon quelles logiques, avec quelles implications. Les débats et solutions préconisés par chacun deviennent exemplaires des problématiques fréquemment à l'œuvre dans les écomusées quand se rencontrent des visions différentes portées par des professionnels des musées et des bénévoles investis dans la défense et la mise en valeur de leur pattimoine. Si les conflits sont généralement vite dépassés, les professionnels finissant le plus souvent par imposer leurs vues, les rapports sont plus complexes dans le cas étudié ici, les professionnels ne résidant pas sur place. Les rapports de pouvoir s'en trouvent modifiés et la normalisation des initiatives locales selon les critères professionnels fortement ralentie. Cette situation en fait un terrain privilégié pour le sociologue désireux de se pencher sur les mécanismes d'appropriation, de transfert et d'institutionnalisation. En effet, ce qui est habituellement occulté et passé sous silence éclate ici plus ouvertement. Car si le cas est exemplaire, il n'est pas marginal ou exceptionnel, mais seulement explicite. La prise en main par d'autres acteurs lors de la professionnalisation conduit toujours à des crises, cas récurrent clans les écomusées.

\section{DES POINTS DE VLE DIVERGENTS}

Si les Amis de l'Écomusée voient dans le projet de rénovation une chance d'accroitre les activités et l'influence de l'association, et possèdent leurs visions de lavenir du site, de son développement et des améliorations à apporter, ils ont des difficultés à en formuler une conception. claire, et à l'exprimer dans un document. Surtout très attachés au travail déjà mené, ils ne peuvent envisager des changements radicaux et militent plutôt pour la continuité. Ils proposent des actions limitées, peu onéreuses, dont ils pourraient quasiment avoir la maîtrise d'œuvre. Toute transformation de l'existant apparaît comme une négation du travail anté-

rieur; s un pre en acci le chas déclenı qui s'e. leur fa muséo bâtime comme $\AA$ vaste accueil figure audiov correct représe chauffe gences régulé. dations véritab musée aussi d où les trouve gences conver norme tiques

LA THE vité pr teur $\mathrm{O}$ : Sont 1 consor mais : sur ce serré démai local : lent $\mathbf{r} \epsilon$ gie co relient comm 
rieur, surtout quand l'idée est proposée par autrui. Ainsi refusent-ils dans un premier temps les modifications avant de parfois se les approprier et en accepter l'éventualité. C'est surtout le fait de ne pas être moteur dans lé changement qui est vécu comme un danger de dépossession et qui qui s'est réalisé de concret de et d'autodéfense. Jusqu'à présent, tout ce leur fait. Une rénovation globale dusible matériellement, sur le site a été de muséographes et réalisée par des architectes orchtrée par une équipe de bâtiment, avec des financements extérieurs, voire européens, est vécue
comme une agression.

A la demande de la municipalité, les professionnels travaillent sur un vaste projet de rénovation de l'équipement. Afin de le rendre moderne, accueillant, capable de correspondre aux critères actuels, c'est-à-dire où figure un espace d'accueil, des lieux de repos, des salles pédagogiques et audiovisuelles, des services tels qu'une boutique, un parking ou des toilettes correctes. Bref, ce que le public s'attend à trouver dans un musée, selon les représentations qu'il s'est forgé dans les grandes institutions. Le musée sera chauffé, ceci pour le confort du visiteur, mais aussi pour répondre aux exigences de la conservation préventive, c'est-à-dire où le climat puisse être régulé. Les objets seront protégés contre le vol mais aussi contre les dégradations diverses menaçant les collections. Par la nécessité de disposer d'une véritable réserve, au sens le plus strict où l'entendent les professionnels de musée, on aborde l'autre aspect du programme. Car évidemment il s'agit aussi de modifier la présentation et le discours tenu sur les collections, point où les conflits surgissent. Sur le premier volet, l'association locale ne se trouve pas vraiment en désaccord et admet rapidement les nouvelles exigences, mêne si elle en discute les modalités. En revanche, sur ce qu'il est convenu d'appeler le Projet Culturel et Scientifique, selon les nouvelles normes institutionnelles, c'est-à-dire sur le contenu, le propos, les politiques mises en ceuvre, les choses sont plus délicates.

\section{LA THÉMATIQUE DU LIEU}

Pour les professionnels, l'écomusée centrera son discours sur l'activité principale, et rendra compte de la vie locale à partir de ce fil conducteur organisateur des échanges locaux. Un concept organise l'ensemble. Sont mis en avant les conditions de production, de diffusion et de consommation de ce produit particulier, des circonstances techniques mais aussi économiques, sociales et culturelles de son développement sur ce territoire et de ses implications. Le propos est volontairement resserré pour se démarquer d'une offre généralisante et confuse. La démarche est scientifique, rigoureuse et n'entend pas sacrifier au folklore local mais tenir un discours ethnologique valide. Les professionnels veulent répondre à des critères et des normes qui sont celles de la muséologie contemporaine. Le discours entend mettre en scène les relations qui relient l'Homme à son milieu, à son environnenent. Les objets viennent comme support d'expression des réseaux de relations. Les aspects tech-

$\frac{87}{\text { LE DFVENR I'LN ÉCONUSET }}$


niques et historiques, mais aussi sociaux, économiques, culturels sont privilégiés. Depuis le départ, l'association locale a un autre projet: celui de montrer de manière exhaustive plutôt que de démontrer. Il n'y a pas un centre autour duquel s'organise l'exposition mais une pluralité d'activités juxtaposées. D'un côté un musée plutôt monothématique, bien qu'offrant une lecture anthropologique du thème décliné, selon la tendance muséologique des années quatre-vingt-dix, de l'autre un écomusée exhaustif et généralisant voulant exprimer l'âme d'un pays, dans toutes ses composantes, à la façon dont de nombreux musées d'arts et traditions populaires l'ont proposé dans les années soixante-dix. Pour le résident, l'écomusée exprime ainsi l'identité de son territoire où chacun doit être représenté d'une façon ou d'une autre. L'association insiste sur sa volonté de mettre en avant une approche globale du patrimoine local, car "tout se tient", façon aussi de mettre en avant les collections d'arts et traditions populaires: activité du forgeron, du vannier, du tonnelier, de l'imprimeur, de la dentellière... Il n'y a guère de limites à cette logique qui recouvre peu à peu toutes les dimensions de l'activité rurale, voire préindustrielle (présentation de petites entreprises locales de transformation). Pour le professionnel ou pour le touriste qui va d'un écomusée à l'autre, la chose est assez répétitive. Ces derniers préféreront voir mettre en avant ce qui constitue réellement une spécificité locale, originale et rarement ou jamais présentée ailleurs. Ce point de vue, souvent exprimé (Prasch, 1992), est inaudible pour l'autochtone pour qui le patrimoine local est incomparáble à un autre, puisqu'il en perçoit l'unicité et les caractéristiques propres. Il est donc possible de souligner une première ambivalence au regard de la définition écomuséale.

\section{LES CONFLITS RÉVÉLATEURS DE REPRÉSENTA'IIONS HÉTÉROGÈNES}

Nous ne pouvons nous attarder ici sur le contenu même du musée actuel et sur le programme proposé en vue de la rénovation, insistons sur les divergences de vue. Polémiques publiques, campagne de pétitions, article de presse locale, menace de procès, demande de référendum local, les conflits vont loin et mettent en scène in fine les conceptions différentes du musée, les représentations sociales qu'en ont les uns et les autres, les missions confiées à un musée, les motivations des acteurs à investir dans tel ou tel projet. C'est la notion même d'écomusée qui est en cause, avec les problématiques qu'il porte, participation et investissement citoyen dans la vie locale, délégation ou dépossession par des instances professionnelles, divergences d'interprétation et d'utilisation des identités et des patrimoines locaux. Car chaque partie utilise les références à ses propres fins. L'association de l'écomusée n'ayant aucunement le monopole d'une parole vraie, parce qu'actrice et initiatrice du projet. Elle est porteuse d'idéologies mais aussi de finalités qui ne sont pas que désintéressées et culturelles. Simplement se pose la question de l'utilisation de l'écomusée comme outil d'expression populaire et de respect de cette expression, quelle qu'elle soit. Bref, c'est le problème de la démocratie

qui se 1 scientifi peu gal
de sens
tations c
Un certé
rogènes.
de ceux
prétatior
de term
sées por
du terme
ou prag1
assimilal
proches
tives prit
cès n'a 1
d'un éco
qui se ré
l'éconus
nelles di
intéressa
caractérí
point de
mutation
statuts,
frques, q
phique. I
sées ous
de théma
un lieu
écomusét
ner aussi
quer les '
qui a été

L'ÉCOMUSÉ

Geor mettre er 
qui se trouve finalement posé et opposé à un discours éventuellement scientifiquement, éthiquement ou techniquement plus cohérent.

\section{QUELLE DÉFINITION \\ D O N N E T - O N \\ D'UN ÉCOMUSEE? \\ I}

de terme d'écomusée est aujourd'hui quelque peu galvaudé par des utilisations abusives mais aussi par les glissements de sens dont il a fait l'objet. Il faut, pour prendre en compte les représentations que s'en font les uns et les autres, rappelet sa définition d'origine. Un certain nombre de divergences proviennent de représentations hétérogènes. Il y a d'abord les approches diverses et souvent contradictoires de ceux qui ont théorisé la démarche écomuséale. Il y a ensuite les interprétations divergentes, les glissements sémantiques et les récupérations de terme, qui font qu'une extrême diversité existe. Beaucoup d'écomusées portent aujourd'hui le nom sans incarner l'esprit originel. Usurpation du terme ou nécessaire évolution des concepts, selon que l'on est puriste ou pragmatique, il clemeure qu'un même terme qualifie des réalités non assimilables. D'autres ne portent pas le nom d'écomusée, mais sont phus proches de la mission confiée par les pères fondateurs. Que des initiatives privées tentent de récupérer un terme qui rencontre un certain succès n'a rien de surprenant et il suffit de réaffirmer les principes mêmes d'un écomusée pour inviter à faire la distinction entre l'authentique et ce qui se révèle être de l'ordre de l'imposture. Ainsi, l'écomusée du miel ou l'écomusée de la chèvre n'ont guère de sens au regard des missions originelles du terme. La distinction devient plus délicate et autrement plus intéressante à interroger quand ce sont les définitions mêmes de ce qui caractérise un écomusée qui évoluent à l'intérieur d'une structure au point de la métamorphoser. Ainsi nombre d'écomusées ont opéré une mutation, tant dans le mode de gestion interne, le fonctionnement, les statuts, les interlocuteurs, les actions culturelles et les projets scientifiques, que dans les principes de médiation et de valorisation muséographique. Leurs publics ont également changé. Demeurent-ils des écomusées ou sont-ils devenus des musées comme les autres, toutes spécificités de thématiques mises à part? Quelles particularités permettent d'identifier un lieu comme un véritable écomusée? Vaut-il mieux parler de postécomusée? Il convient de revenir aux théories fondatrices. On peut imaginer aussi que les définitions demeurent volontairement floues pour masquer les évolutions vis-à-vis des principes fondateurs et pour occulter ce qui a été modifié des rapports de pouvoir dans l'institution.

\section{L'ÉCOMISSÉE, REFLET D'LIN TERRTTOIRE}

Georges Henri Rivière (GHR) définit l'exposition comme "l'action de mettre en valeur, à destination de tout public, un ensemble de biens 
mobiliers, immobiliers ou fongibles, selon un programme précis et dans un espace déterminé " (Rivière, 1989). Surtout, le projet de GHR est de relier des objets, de montrer les rapports de nécessité, d'étudier les objets dans la logique de leur environnement. Il ne s'agit certainement pas de simplement montrer des objets pour eux-mêmes mais d'exprimer à travers eux une culture et les relations des habitants à un territoire. Sans développer ici les différences entre la muséologie telle que l'exprime GHR et ce que sera ensuite la nouvelle muséologie, nous pouvons insister sur ce qui les rapproche face à une exposition sérielle digne du musée taxinomique. Héritiers des classements systématiques en vue d'établir des typologies, chères à l'appréhension scientifique du xixe siècle, les musées ont d'abord développé des approches sérielles les conduisant à s'apparenter aux présentations proposées par les collectionneurs. Les petits musées conçus par des bénévoles au sein d'une association de valorisation du patrimoine local se réfèrent le plus souvent à cette conception du musée, liée aux représentations traditionnelles qui lui sont attachées. Or l'intention de GHR, et a fortiori celles des muséographes qui suivront, vise à s'émanciper de cette filiation et à proposer une muséologie de discours, où l'objet est un élément nécessaire mais non suffisant à l'élaboration d'un contenu. Si GHR demeure encore attaché à l'objet intrinsèque comme porteur de sens, la nouvelle muséologie s'en émancipera au point de parfois faire disparaître l'objet au profit d'une affirmation des points de vue. Quoi qu'il en soit, les deux approches sont en accord sur le refus de sacraliser l'objet pour lui-même. Dans la lignée de Claude Lévi-Strauss, qui souhaite un musée qui n'amasse pas les objets comme des restes desséchés dans un herbier mais qui soit un outil pour comprendre et analyser des modes d'existence (1967), les conceptions nouvelles visent à exprimer au mieux des situations. "Si l'objet n'est pas cette voie royale d'accès à la complexité sociale, s'il n'ouvre pas sur la totalité économique, sociale, symbolique, religieuse, technique et esthétique, alors il n'est qu'un témoin desséché" (Mairot, 1992). Nous pouvons noter ici une première différence avec les conceptions des bénévoles que nous avons interrogés, pour qui les objets sont porteurs de sens et de significations et se suffisent par eux-mêmes. Ainsi seront-ils présentés en situation d'usage, au mieux au moyen cle dioramas ou de classements thématiques, mais le plus souvent commentés indépendamment les uns des autres, notamment lors des visites guidées.

GHR usait de la métaphore du miroir pour exprimer la fonction de l'écomusée comme reflet d'un territoire, de ses activités et de ses habitants. Or Philippe Mairot note, avec raison, que le rôle des musées de dire l'identité ou de la préserver est mis en cause dès lors que celle-ci n'est plus perçue comme immuable, telle une donnée de nature, mais comme une figure incessamment reconstruite, sujette à élaboration permanente, en proie aux mutations, aux évolutions, production même des réinterprétations (Mairot, 1997). Ainsi les identités ne sont plus des données objectives, il faut se pencher sur les conditions concrètes de leur production. Le sentiment d'identité collective, de "nous", est une conscience de soi mouvante, sans cesse réinterprétée, sujet à un travail de réécriture perpé-

tuel.

sans $\mathrm{l}$

des

qu'ell

gemes

dans

vision

comn:

réacti

par e

1992).

échan

située

que l'

Patricl

facette

de va.

celui ,

qu'il a

le rôle

distan

Cette

sujet $c$

un tes

symbc

toire

une d

cherct

de sor

de lir.

récurs

négoc

mité c

révéle

objets

ficatio.

ils térr.

place

$\operatorname{long} \mathrm{f}$

pas lá

parce

sélecti

le mu.

ajouté

coffre-

L

c'est-à

voit $p$ 
tuel. Les sciences humaines ont montré qu'il n'existait pas de sociétés sans histoires et que l'Histoire était toujours relative à des lectures, donc à des points de vue, des reconstructions. Les sociétés traditionnelles, qu'elles soient européennes ou non, ont toujours été en proie aux changements. C'est l'accélération des modifications et des bouleversements dans les sociétés industrielles, depuis deux siècles, qui a donné une vision arrêtée et glacée de certaines sociétés. Le désir de figer l'identité comme une entité intemporelle correspond aussi à une vision politique réactionnaire du corps social. Jean-Claude Duclos, Jean-Yves Veillard ont, par exemple, mis en garde sur les dérives possibles (Duclos, Veillard, 1992). Les recherches montrent que le local est en fait inséré dans des échanges incessants qui ramènent à l'universel, que les traditions sont situées historiquement, que les frontières sont le plus souvent floues, bref que l'identité est mouvante, plurielle, changeante, comme le note aussi Patrick Prado (1995). Le même est multiple, et dispose de différentes facettes. II s'agit par conséquent de s'accorder sur l'identité qu'il convient de valoriser. Le groupe a tendance à se présenter sous son meilleur jour, celui qu'il estime intéressant. Si chacun mythologise son histoire dès lors qu'il a quelque chose à transmettre, comme le rappelle Marc Augé (1992), le rôle du lieu patrimonial est au contraire d'engager à la réflexion et à la distance critique et non de participer à l'élaboration d'une idéologie. Cette dialectique de l'enfermement et đu dépassement se trouve être le sujet de différends entre chercheurs formés à appréhender objectivement un territoire et une population sensible prioritairement à la dimension symbolique, à la charge émotive que recèle tel objet, tel fragment d'histoire de vie, tel monument, constate Jean-Michel Barbe (1985). Devant une divergence d'interprétation, quel discours doit prédominer? Celui du chercheur qui use de son autorité scientifique, du conservateur du haut de son pouvoir institutionnel, de l'usager dont la connaissance est vécue de l'intérieur? L'exemple n'est pas un cas d'école, il s'agit d'un problème récurrent dans. les écomusées, même s'il est le plus souvent résolu sans négociation par l'affirmation du pouvoir de l'un sur les autres. La légitimité des choix et du sens que portent les objets et que le musée doit révéler peut dès lors être mise en cause. Il ne suffit plus de faire cire aux objets ce qu'ils recèlent de leurs anciennes appartenances, de leurs significations, de leurs usages, de leurs symboles, etc., mais aussi de ce dont ils témoignent en tant qu'objets choisis par des acteurs en vue de prendre place dans une opération symbolique ou sociale. L'image du miroir a fait long feu, poursuit Philippe Mairot, pas seulement parce que le signe n'est pas la chose, parce que l'objet mort représente un autre ailleurs vivant, parce que le représentant n'est pas le représenté, mais parce que le miroir sélectionne une partie du réel, le déforme, le découpe. Surtout parce que le musée ajoute autre chose de plus au réel. "Il est une chose de plus ajouté au monde": le musée ne représente pas le réel, il n'est pas un coffre-fort recelant des reliques.

Dans ce paradigme, le musée devient le lieu même de l'uchronie, c'est-à-dire d'une relecture particulière de l'Histoire telle qu'on souhaite la voir présentée. Une utopie appliquée à la mémoire. Une tension s'exerce 
alors entre un passé mythique et un avenir utopique, où l'écomusée doit définir son domaine d'action, selon François Hubert (1985). Dès lors, on peut interroger la pertinence des points de vue et pour ce qui nous intéresse ici leurs divergences. Le conservateur devient ce démiurge qui fait surgir du sens, un auteur qui compose une partition, propose une lecture, fait surgir une interprétation. Le conservateur "n'est plus dès lors un discret porte-parole, mais un créateur devant une page blanche", selon Philippe Mairot (1997). Or, à ce niveau s'opère le glissement dans la théorie écomuséale. Sous prétexte que l'identité n'apparaît pas comme unitaire, se donnant comme instituée et immédiate, mais comme produite et reconstruite, le professionnel n'en est plus le porte-parole, mais l'interprète, si ce n'est l'inventeur. Si la critique de GHR sur la conception de lidentité et sur sa traduction littérale au travers de l'objet peut et doit être conduite, en revanche peut-on oublier que la théorie écomuséale stipule aussi d'être le porte-parole d'une population? On pourrait imaginer que le conservateur demeure humblement l'instriment au service d'une population désireuse d'exprimer son interprétation de l'identité, qu'elle sait par ailleurs relative, partielle et partiale. On pourrait imaginer qu'il laide dans son cheminement, sans pour autant la devancer. Or on comprend très bien que le conservateur préfère découvrir une forme narrative et proposer sa lecture pour faire surgir un sens nouveau. La position est plus gratifiante et plus stimulante. La réflexion en vient donc à établir une relation duale entre le concepteur-créateur et le public. Cependant, après avoir présenté le conservateur comme l'être tout-puissant qui dispose et propose sa création, l'auteur revient sur la contradiction en relativisant sa proposition. Se rappelant avoir oublié la population en chemin, il envisage le public comme partenaire, voire comme co-auteur, avec des experts au service des amateurs. Et, comme pour se dédouaner, l'auteur de rappeler la formule d'Hugues de Varine en 1973 pour qui "la communauté tout entière constitue un musée vivant dont le public se trouve en permanence à l'intérieur. Le musée n'a pas de visiteurs, il a des habitants. Toutefois, on ne voit pas comment s'opère la transition du conservateur démiurge au conservateur modeste. Lád notion de coauteur incarne davantage le partage entre le conservateur et les experts extérieurs que le travail avec les volontés locales. D'autant que la mission de celui-là demeure de faire passer des discours du domaine ésotérique du spécialiste au domaine exotérique. Dans une posture pédagogique, qui adopte des techniques de présentation qui soient des postures de traduction, comment celui qui a le pouvoir de la connaissance peut-il être au service et porteur de l'expression de ceux à qui il est censé s'adresser? En réalité, la contradiction se lève dès lors que l'on comprend que le public n'est plus le même. La population a été évincée au profit du développement touristique. "Habitants ou clients? Figures du visiteur dans un paysage changeant", s'interroge Philippe Mairot en reprenant le terme de l'écomuséologie. Et l'auteur remarque justement que la logique de recherche du client pourrait bien amener à un changement d'institution, ceci en dépit de la persistance des noms et des déclarations d'intentions. 
ée doit ors, on us intéqui fait lecture, un disselon la théone unifuite et lintertion de loit être stipule her que - d'une qu'elle ser qu'il on com2 narraa posidonc à public. ut-puisntradicpopulacomme pour se en 1973 dont le de visispère la sste. La ervateur J'autant ouxs du ans une tion qui jir de la a ceux à dès lors Ilation a ants ou nterroge lauteur rait bien rsistance

\section{ACTEURS OU TÉMOINS? L'ÉCOMUSÉE COMME LIEU D'INITIATIVE LOCALE}

Lié à la décolonisation et à la contre-culture, l'écomusée a porté cette formidable utopie de proposer aux habitants d'être acteurs de l'élaboration de leur Histoire. "C'est un instrument qu'un pouvoir et une population conçoivent, fabriquent et exploitent ensemble." (Rivière, 1971-1980.) Car l'écomusée n'est pas seulement un lieu au service de la population, il s'agit d'abord d'un lieu conçu par cette population. L'idée est d'aller bien au-delà d'une coopération avec les habitants pour que ceux-ci soient réellement initiateurs et acteurs du lieu. Un écomusée ne saurait être imposé de l'extérieur, il s'agit d'une initiative locale, écrit Hugues de Varine. Pour André Desvallées (1992, p. 27), il ne s'agit pas seulement d'animer le musée et de l'ouvrir à un public élargi, mais que ce public s'approprie, qu'il prenne l'initiative des actions et de faire de tout son patrimoine le fond du musée. L'ethnographie s'applique à soi-même: sa culture d'appartenance devient digne d'intérêt, valorisée, reconnue, exhibée. Toute chose traduit un réseau de significations, témoigne d'une historicité et révèle des rapports sociaux. Mais il ne s'agit pas de s'enfermer, la culture des autres devient enjeu et digne d'intérêt pour elle-même, par ce qu'elle révèle de ma propre culture, parce que finalenent elle pointe les interrelations. Ce projet de construction et de présentation d'une Histoire devant mêler les motivations des acteurs quelle que soit leur origine sociale, professionnelle, culturelle. L'échange et le va-et-vient entre les propositions des uns et des autres permettent d'élaborer une Histoire commune. Ainsi sont mêlées les voix des universitaires et des chercheurs, des spécialistes, aux voix anonymes des locaux. Force est de constater que les conflits ont souvent éclaté sur les différends entre amateurs, associations locales, chercheurs, ethnologues, muséographes, conservateurs. Les uns et les autres n'ayant pas les mêmes référents et ne partageant pas les mémes conceptions de ce qui doit être valorisé, et des formes de
médiation à retenir.

Dans le cas de l'écomusée étudié, les instigateurs entendent continuer à concevoir, gérer et développer le lieu selon leur conception. Face aux professionnels, ils défendent les valeurs qu'ils souhaitent exprimẹr au travers des présentations. Quoi que l'on puisse penser de la pertinence et des contenus idéologiques ou mythologiques de cette culture locale reconstruite, demeure la question de la prise de décision. Selon que l'on valorise, dans la théorie écomuséale, l'affirmation d'un contenu scientifrque exigeant, ce qui serait plus proche de Georges Henri Rivière, ou limplication des acteurs, ce qui est privilégié par Hugues de Varine, on se place dans des perspectives divergentes. Faut-il affirmer des choix dictés par une logique scientifique, ce qui conduit à mettre en avant la cohérence des contenus, ou bien faut-il d'abord penser l'écomusée comme un instrument d'implication et de transformation des rapports sociaux locaux, ce qui conduit à valoriser les acteurs? Ces deux perspectives, si elles sont présentées comme convergentes dans la théorie de l'écomuséologie, se révèlent le plus souvent divergentes sur le terrain. La prédomi- 
nance d'une parole autorisée, celle des experts, sur les contenus, conduit irrémédiablement à mettre les acteurs locaux en position d'objets et de retrait.

UN LIEU DE PARTICIPATION POPULAIRE ET' UNE RESSOURCE POUR I.E DÉVEIOPPEMENT LOCAL

"L'écomusée est un instrument de participation populaire à l'aménagement du territoire et au développement communautaire", écrit Hugues de Varine ( $\mathrm{HdV})^{39}$. C'est un instrument de démocratie directe, qui permet l'échange, le débat, la confrontation d'idées pour parvenir à des solutions collectives. Lieu d'expression et de prise en main de sa destinée par un collectif qui entend maitriser de façon libre et responsable les problèmes posés au niveau local. L'écomusée a d'abord une fonction d'instrument de développement communautaire, et ressemble plus en cela à une coopérative, à une maison du peuple ou à un syndicat autogestionnaire qu'à un musée traditionnel. L'objectif de préserver et de mettre en valeur un patrimoine devient une des missions d'un lieu dont la visée est bien plus large. La gestion par un collectif de représentants d'associations vise à asseoir une conscience politique d'implication citoyenne qui repose sur la connaissance de ses origines et de son devenir. Pour cela, l'écomusée est un lieu d'auto-analyse de la communauté sur elle-même et l'instrument d'une conscience critique collective au service du développement communautaire. La population locale est donc non seulement objet mais sujet de l'institution, non seulement public mais acteur de l'action et de l'animation. Les auteurs de Territoires de la mémoire écrivent ainsi que le musée-forum est un lieu d'expression de la démocratie.

L'analyse des discours des bénévoles, acteurs de l'écomusée, conduit davantage à repérer les relents nostalgiques et passéistes, voire les propos réactionnaires d'un ruralisme exacerbé ou même d'une démagogie populiste, que les effets d'une citoyenneté ouverte, généreuse et critique d'elle-même. Ainsi la théorie généreuse de l'écomuséologie peut-elle être relativisée ou repensée. Faut-il construire comme parole autorisée une parole produite par les acteurs eux-mêmes? Le scientifique, selon une épistémologie de la rupture, est comptis comme celui qui désenchante, qui ne reprend pas nécessairement en chœur les idées locales autoproclamées, qui ne vise pas à l'approbation, à la célébration ou à la commémoration. Il est plutôt là pour cénoncer le récit d'un passé radieux et de ses mythes fondateurs. Mais cette perspective bourdieusienne, veut-elle dire que la recherche serait par nature l'affaire du spécialiste et que la recherche menée de façon autodidacte tiendrait lieu de recherche déclassée, de souls-recherche? N'est-ce pas une façon que les chercheurs ont de s'approprier la recherche, alors que de nombreux savoirs ont de tous temps été produits par des amateurs? Y a-t-il opposition entre le savoir vaai du scientifique et le savoir faux, le sens commun, la croyance de l'indigène? On sait que cette dichotomie bachelardienne est un des mythes de la science positiviste. C'était justement un des enjeux de l'éco- 
muséologie que de la remettre en cause, même si les positions de GHR sont parfois ambiguës à ce sujet. Par ailleurs, la parole "vraie" de l'expert peut être jugée impérialiste, être elle-même relativisée et sujette à subjectivisme. Les critiques menées par les différents communautarismes sur les savoirs produlits à leur encontre l'expriment suffisamment. Un groupe parle d'un autre au nom de la vérité. Par ailleurs, lobjectivité et la neutralité recherchées dans une position d'extériorité ne produisent pas seulement une fiction scientiste, elles sont également le lieu d'un savoir froid. Contre cela il faut, selon Marc Guillaume, que se rassemblent, dans un même mouvement, le travail de la mémoire in vivo et le travail sur la mémoire, in vitro. Et Philippe Mairot de noter qu'il s'agit alors d'inventer la fiction d'un passé commun de la rencontre de ces deux sources sans quoi la politique du patrimoine n'est qu'une forme vide. Toutefois, si le savoir du scientifique se construit contre le sens commun, comment concrètement faire se rejoindre deux appréhensions contradictoires? Comment présenter une perception tronquée de la réalité et le savoir vrai produit par l'activité de connaissance? Il faut renoncer soit à la lecture positiviste de l'élaboration du savoir, soit à la présentation commune d'une lecture de l'Histoire contenue dans l'écomuséologie. L'expert ne peut dévoiler la vérité à ses objets et être à leur service. De cette contradiction naissent les changements de publics dans l'institution. Comment concilier les volontés locales de construction mythologique et de transformation de l'objet en relique et l'approche de la nouvelle muséologie qui prétend transcender l'objet pour produire du sens? Le pari de l'éconuséologie devrait être plutôt d'éduquer à la recherche en développant l'analyse critique des acteurs, bref de produire des mentalités de chercheurs, sans quoi l'approche locale ou bien est niée par le professionnel, ou bien devient une illusion sociale. La contradiction entre ces deux discours se résout généralement par l'imposition d'un semblant de consensus affirmé par celui qui représente la parole autorisée.

\section{LA PLACE ET LE RÔLE DES EXPERTS}

Si l'écomusée est clairement défini conme à l'écoute de ses habitants, sa conception et surtout son mode de fonctionnement révèlent certaines ambiguités. Pour Hugues de Varine (HdV), l'écomusée est un contre-pouvoir. Aussi est-il conçu et géré par les locaux. Le professionnel doit être au service des habitants et s'effacer derrière leur décision. Pour GHR, dans une logique plus institutionnelle, il faut approcher une population pour la servir, toutefois il faut maintenir un équilibre raisonnable entre pouvoirs de l'autorité de tutelle et aspirations de la population concernée. Contrairement à HdV, GHR ne soumet pas en dernier recours l'expert à la population. De cette divergence, qui finalement révèle des paradigmes hétérogènes, naîtront les divisions qui conduiront à réaffirmer la prééminence du discours autorisé. Les deux positions conduisent de toute façon à des dérives que l'on peut énoncer rapidement. On peut reprocher à GHR de minimiser les rapports de pouvoir ou de penser trop 
naïvement que les pouvoirs puissent ceuvrer à leur relativisation. En effet, si l'écomusée est véritablement ce contre-pouvoir, lieu de proposition et de prise en main citoyenne de son destin, le contrôle de cette instance, qui pourrait être réellement démocratique, devient d'autant plus urgent pour les pouvoirs locaux. Elle représente un risque certain de perturbation politique. La réussite de l'expérience de l'écomusée aurait nécessairement une charge subversive pour les institutions en place, au fonctionnement si rarement démocratique. La place est donc rapidement investie par des motivations peu désintéressées. Parce que l'initiative crée du lien social et du dynamisme, les élus sont enthousiastes tant qu'ils peuvent s'en prévaloir. Le premier consensus autour de la valorisation patrimoniale s'effrite quand la prise de parole dans l'association amène les acteurs à utiliser leur droit de parole dans d'autres domaines. "L'élu se sent doublé par ces bénévoles qui se mêlent de tout. Le consensus s'effrite peu à peu. Il devient alors urgent de confier la gestion de la plateforme culturelle à des "professionnels"." (Cotton, 1998, p. 32-33.) Au nom d'arguments divers: compétences scientifiques et techniques, financements, sécurité des collections ou du public, survie de l'institution, reprise en main d'activités déclinantes, crédibilité de l'institution, mise aux normes muséographiques, le professionnel intervient, qu'il soit conservateur, muséographe, ethnologue... La dépossession se manifeste alors implicitement. "Et l'ethnologue est alors là pour justifier dans son langage disciplinaire ce qui n'est au fond qu'un rapt de pouvoir. Il y a là un retournement: l'ethnologue, dans un premier temps, a aidé la population locale à reconnaître ses savoirs et à mettre en majesté sa culture et, dans un deuxième temps, il aide les institutions à la récupérer" (Ibid.). Les exemples sont nombreux où les associations moribondes de bénévoles vieillissants sont remplacées par des professionnels avec les changements d'orientation que cela implique. Mais sont également fréquentes les situations où une association bien vivante et active se voit dépossédée par une professionnalisation à laquelle elle résiste et qui finit par avoir raison d'elle. On pourrait multiplier les exemples car presque tous les écomusées ont les leurs. Les conservations départementales, plus éloignées des instances locales, et donc moins impliquées, bénéficiant d'une force de frappe importante notamment au niveau économique, vont parfois être ces instruments d'institutionnalisation et de dépossession. L'opération de marginalisation puis d'élimination des volontés locales bénévoles se fait souvent au cours d'une opération de rénovation, qui impose des procédures lourdes, des compétences techniques de plus en plus complexes, et des réorganisations de forme et de contenu. Le cycle se referme quand les seuls spécialistes sont délégués à la gestion de la mémoire et à sa mise en vitrine. Ce qui était appropriation collective devient délégation.

Dans le transfert de la gestion du musée au profit des professionnels se jouent des rapports au patrimoine qui n'ont pas la même signification. Derrière l'apparence de continuité, c'est le rapport à la mémoire qui est assumé ou délégué. La culture locale est alors mythifiée autrement, et échappe à nouveau à ceux qui devraient logiquement en être les gardiens

el les

du $\operatorname{Re}$

locale:

n'aurc

assimi

đépos

progrc

l'inves

aux $\mathrm{p}$

rel. $\mathrm{Br}$

terrain

doit $\hat{e}$

structi

musée

acteur:

demer

C

cité d'

aussi

comm

admin

consig

sion d

damer

de qu

tique:

avertit

l'intéré

ne do

qui se

fait dit

sident

musée

lective

par le:

chacus

bléma

par l'a

savoir

intervi

peuve

P.

volont

profes

contra

cipe à

son es

acteut 
n. En effet, sposition et -e instance, sus urgent e perturbait nécessai$\mathrm{u}$ fonctionsnt investie rée du lien is peuvent $n$ patrimoamène les 3. "Liélu se consensus de la plate3.) Au nom $s$, finance'institution, Ition, mise qu'il soit : manifeste - dans son ir. Il y a là la populaculture et, er" (lbid.). : de béné: les chanfréquentes épossédée par avoir e tous les plus éloiiant d'une vont paron. L'opéales bénénui impose s en plus - cycle se ion de la collective

essionnels nification. ire qui est ement, et $s$ gardiens et les dépositaires. Ancien président de l'association des amis du musée du Revermont, Jean-Pierre Cotton s'interroge à ce sujet: "Les populations locales, passées les premières années, éliminées de la gestion des projets, n'auront plus droit qu'à la reconnaissance formelle. L'ethnologue sera assimilé à ce regard extérieur, intéressé et récupérateur synonyme de dépossession". Sous prétexte d'objectivation scientifique et de rigueur, le programme scientifique sera construit par des spécialistes, en dehors de l'investissement des intéressés. Il est dès lors difficile de faire une place aux populations pour qu'elles s'investissent ensuite dans le projet culturel. Bénévoles et professionnels n'ayant pas la même connaissance du terrain, mais surtout pas les mêmes représentations préalables de ce que doit être le lieu, ne vont pas promouvoir le même développement de la structure. Ainsi la fabrication de consensus, spécialité s'il en est des musées, cache des prises de paroles et des invitations au silence. Les acteurs des sujets présentés sans eux oublient dans le même temps de demeurer des usagers d'un éconusée devenu musée pour d'autres.

Clairvoyant contre les dérives susceptibles de survenir, HdV (1978, cité d'après Vagues, 1992, p. 483) mettait en garde: "Un écomusée peut aussi être un instrument de mainmise d'un groupe de technocrates sur la communauté: fonctionnant alors comme un instrument de paternalisme administratif, il est voué à l'explicitation et à la communication de consignes simples, pré-arrangées, qui donneront à la population l'impression de décider de son développement alors qu'en réalité les choix fondamentaux sont faits en dehors d'elle. "La communauté sera alors le jouet de quelques-uns qui se serviront de la structure comme leurre démocratique alors que les véritables instances décisionnelles lui échappent. HdV avertit que le danger d'une prise de pouvoir et d'un détournement de l'intérêt collectif peut provenir de l'extérieur ou de l'intérieur. L'écomusée ne doit pas être aux mains d'une personnalité très forte et directive, ce qui serait contradictoire avec l'esprit même de l'écomusée, que ce soit le fait d'un directeur, d'un conservateur charismatique, d'un maire, d'un président ou d'un membre d'une association locale qui s'appropriait l'écomusée. En principe, dans un écomusée, les programmes sont décidés collectivement et négociés, tant au niveau de la conception que des actions par les usagers. "L'avenir de tous étant conditionné par la manière dont chacun saura poser les problèmes collectifs, il est essentiel que cette problématique ne reste pas le domaine réservé de fonctionnaires délégués par l'autorité centrale ou d'élus mis en situation de notables détenteurs du savoir et du pouvoir" (lbid.). Les techniciens et divers professionnels interviennent comme prestataires de service, comme conseillers; ils ne peuvent pas décider.

Pour HdV, la notion de public est dès lors impropre ou marginale. La volonté d'élever la fréquentation, qui semble être le nouveau credo des professionnels, devrait donc être problématisée: n'est-ce pas un élément contradictoire avec la mission des écomusées? La population qui participe à l'élaboration et au développement du site, et au travers de lui de son environnement, est moins à percevoir comme public que comme acteur. La notion de partenaire constitue déjà un premier glissement de 
sens et d'investissement dans le lieu. Ce n'est pas seulement la gestion et le fonctionnement, les orientations, les sujets de recherche et les discours à tenir dans l'écomusée qui sont décidés par le collectif, lequel, tout en tenant compte de l'avis de l'expert, a néanmoins le dernier mot, il s'agit d'inventer de nouvelles formes d'expression. Ainsi HdV postule que des formes nouvelles seront dégagées, une sorte d'invention populaire du quotidien, mais aussi des techniques muséographiques à mettre en cuvre. De sorte que c'est la muséologie elle-même qui se verra réinventée, ou du moins qui connaîtra des apports nouveaux. "L'écomuséologie ne peut donc plus être laffaire des muséologues et des spécialistes de sciences humaines, elle doit d'abord associer les responsables du territoire et de la communauté qui seuls sont susceptibles d'inventer les formes nouvelles que l'action de l'écomusée devra prendre" (Ibid.). On pourra reprocher à cette vision les influences idéologiques du marxisme qui espérait une science refondée sur un matérialisme conduit par des principes non bourgeois. Il s'agit ici de croire à des voies nouvelles inventées par une pratique collective du pouvoir. Et s'il convient de tempérer l'enthousiasme de l'époque au vue des expériences menées, on peut néanmoins convenir que les mouvements communautaires ont effectivement inventé d'autres formes de rapports sociaux et donné lieu parfois à des expressions nouvelles de créativité.

Le reproche majeur que l'on peut formuler à la thèse d'HdV réside dans la soumission à une sorte de dictature du local. Trop attentif peutêtre au phénomène de récupération et de détournement, dans la mouvance foucaldienne des années soixante-dix, HdV se place dans une perspective libertaire qui aboutit finalement à faire de la parole proférée de l'intérieur la seule parole autorisée. La logique communautariste est ainsi poussée à son terme. Les professionnels doivent faire partie intégrante de la communauté en s'impliquant dans la vie et lhistoire locale au point d'en devenir un élément parmi d'autres. Leur implantation et leur assimilation sont la règle (Ibid.). Il faut bien évidemment replacer dans le contexte de l'époque ces prises de positions qui peuvent apparaître aujourd'hui dangereuses. Ainsi, dans le cas de l'écomusée étudié dans cet article, l'opposition se cristallise contre la conception des technocrates, c'est-à-dire de ceux qui ont pensé le local en dehors de son territoire. La culture de la ruralité n'est sans doute pas ici sans importance sur la perception de l'extérieur. Les bénévoles insistent sur le fait que le projet muséographique, le contenu de ce que l'on va dire sur le pays ne peut être dit que par "des gens du coin", et non pas par des professionnels habitant hors du territoire, fussent-ils spécialistes. Car il ne peut $y$ avoir de meilleurs spécialistes du local que les locaux. La communauté a nécessairement un point de vue plus pertinent sur elle-mêtme et sur ses origines que quelqu'un de l'extérieur. Sí les intentions de HdV sont louables et généreuses, elles comportent ce danger de dérives communautaristes ${ }^{40}$. À vouloir relativiser le pouvoir des intervenants extérieurs, HdV prend le risque d'enfermer la communauté sur elle-même. Confier le dernier mot au local, tant pour les choix d'orientation, le contenu des discours que pour les principes et modes de fonctionnement, c'est finale-

ment a

nance $\dot{z}$ une qu préémi: notanur. qu'hétì nelle, 1 lieu. B depuis une pe opposé les pris en elle ces biel

UN LUL

écomu peut $\hat{E}$ mieux figer : sienne tnet d points tion a ouvert les ser tent. I fessior conce! lHom: et l'au mond identit une $a$ n'est $\epsilon$ univel l'intéri lieu di se rat auque mis et "Sauv' mais $j$ conte: cultur 
1 gestion et es discours iel, tout en rot, il s'agit le que des spulaire du mettre en ra réinvennuséologie cialistes de s du terriiventer les (Ibid.). On I marxisme nit par des zlles invene tempérer 3 , on peut t. effectiveu parfois à

IdV réside entif peutus la moudans une e proférée itariste est sartie intéoire locale zntation et it replacer rent appasée étudié des techte son termportance fait que le le pays ne rofessionne peut $y$ munauté a et sur ses HdV sont $s$ commulextérieurs, Confier le ul des disest finale- ment ancrer l'autorité d'une parole dans un terroir. Comme si l'appartenance à une communauté, à un sol, voire à une lignée, à un sang, donnait une quelconque légitimité. C'est in fine l'argument conservateur de la prééminence des traditions et de l'héritage sur toute autre dimension (et notamment de la recherche, de la science, de la culture construite plutôt qu'héritée). Plus que de l'élaboration d'une pensée argumentée et rationnelle, l'autorité provient des ascendants et de son inscription dans un lieu. Bref, c'est l'argument utilisé par tous les mouvements rétrogrades depuis l'Ancien Régime. Alain Finkielkraut (1987) a su montrer comment une pensée culturaliste de gauche rejoignait ainsi finalement ses pires opposants. Les dérives communautaristes, que l'on observe parfois dans les prises de parole des minorités à la recherche d'une légitimité fondée en elles-mêmes, sont alors susceptibles de trouver dans l'écomusée un de ces lieux d'expression.

\section{UN LIEU POUR OUOI?}

Le danger de la tentation nationaliste, ou plus exactement dans les écomusées d'exaltation de la culture régionaliste, existe. La présentation peut être une appropriation et une refondation de son identité pour mieux la redéployer, elle peut être aussi instrumentalisée pour mieux figer le présent et les rapports sociaux, selon une idéologie maurrassienne. Contre cela, le relativisme anthropologicque est nécessaire. Il permet d'ouvrir le local sur des données extérieures, des échanges, des points de vue décalés et de mettre le local en dialogue si ce n'est en situation avec d'autres lieux, d'autres époques, d'autres sociétés. Or cette ouverture proposée par le professionnel ne manque pas de venir heurter les sensibilités de ceux qui entendent exalter l'unicité de ce qu'ils présentent. Le document de programmation muséographique établi par les professionnels, dans le cas de l'écomusée analysé ici, correspond à la conception de la nouvelle muséologie. Il met en avant les relations de l'Homme à son milieu, mais aussi les liens entre l'ici et l'ailleurs, le mêne et l'autre. "Un regard sur l'ailleurs, sur d'autres horizons, vers d'autres mondes". Bref, il affirme une politique de mise en correspondance des identités locales avec les identités multiples. Il décloisonne et propose une conception relativiste de la culture par une mise en perspective, si ce n'est en abîme, de la culture locale vis-à-vis d'une conception globale et universalisante. Il s'agit de mettre en avant des spécificités en les situant à l'intérieur d'une approche anthropologique et transversale. Or il y a tout lieu de penser que cette conception de la présentation de lidentité locale se rattache à un paradigme qui se trouve en profonde rupture avec celui auquel se réfèrent les locaux. Ceux-ci n'entendent pas être "relativisés" et mis en parallèle avec les rituels d'une autre région, moins encore avec les "Sauvages" de Nourvelle-Guinée! L'exemple est extrême et caricatural, mais il exprime finalement le problème profond. L'intérêt d'une mise en contexte et d'une relativisation est évident pour le professionnel féru de culture anthropologique; il est plus insupportable pour l'autochtone. 
Celui-ci recourt au musée pour magnifier une culture locale et mettre en représentation la profondeur et la richesse de ses origines et de son milieu de vie. Il entend célébrer les coutumes locales comme des moments uniques et authentiques. Certes, il s'agit là de mythologies, et les locaux n'en sont pas toujours dupes. Jean-Pierre Vernant posait la question de savoir si les Grecs croyaient en leur mythe. On sait bien aujourd'hui la duplicité qui entoure le plus souvent la croyance. Finalement, c'est l'adhésion aux mythes qui leur permet de fonctionner, la duplicité ne dissout pas l'efficacité sociale. Du reste, "les mises en scène de la vie quotidienne" nous l'enseignent également. Ainsi, le musée est un lieu de construction et de représentation du mythe contemporain. Or le professionnel ne vise pas moins par son travail de relativisation à đémythifier et à transformer en connaissance froide des lieux d'identification, par nature chauds. Dès lors, il n'est pas surprenant que ces musées n'accueillent ensuite que les classes cultivées disposant des codes de lecture appropriés. Il s'agit pour eux moins d'achérer que de comprendre.

IES DIVERGENCES
ENTRE BÉNEVOLES
ET PROFESSIONNELS
ll est assez savoureux de voir les locaux reprendre couramment à leur compte la paternité de Georges Henri Rivière pour défendre leur conception et s'opposer au conservateur qui s'en réclame également. En fait, c'est par les professionnels que l'association a découvert un auteur qu'ils maitrisent certes assez mal mais dont ils ont assez bien perçu la démarche. Les uns et les autres se disputent ains l'héritage au nom de conceptions et de lectures différentes. Les locaux entendent défendre l'esprit des écomusées face aux professionnels qui s'en font occasionnellement les théoriciens. On peut dès lors se demander qui est le gardien du temple? Au travers des arguments opposés au projet défendu par les professionnels nous pouvons cerner les représentations et icléaux muséaux des uns et des autres. Plusieurs points peuvent être repérés dans la dissension: le coût de l'opération, les statuts du futur musée, l'affectation des espaces, les modifications apportées dans les présentations, les discours à tenir dans les salles, les types de médiation retenus. Au travers de ces différents points, c'est plus profondément la destination du lieu, sa fonction sociale qui sont en cause, et au bout du compte la définition de l'écomusée.

\section{LE COUT' DE L'OPÉRATION}

La première raison donnée spontanément par l'association pou s'opposer au projet réside dans le coût de l'opération de rénovation. Le Conseil municipal a voté (à une voix près), sous la pression insistante des professionnels, des institutions partenaires et du renfort du préfet, le pro- 
jet de rénovation pour un montant de plus de 12 millions de francs, L'association propose, quant à elle, un projet de rénovation de moins de la moitié. Or cette enveloppe ne paraît pas crédible aux professionnels, et en particulier à la direction des Musées de France. La commune devrait alors vraisemblablement porter seule cet investissement (ce que lui interdit sa capacité financière), car les partenaires institutionnels se retireraient alors probablement du financement. Du moins menacent-ils de le faire. À première vue l'argument local semble ridicule, puisque le coût communal, et donc l'imposition pour l'habitant, ne sera guère plus élevé dans le premier scénatio alors que les moyens dévolus à la rénovation seront sans comparaison. Il y a tout lieu de penser que l'argument économique est un prétexte mis en avant de façon démagogique pour affoler le contribuable, futur électeur, et pour occulter des raisons plus profondes. La différence entre les coûts d'investissement nécessaires à la création du musée, qui a rassemblé toutes les bonnes volontés locales, et les budgets consacrés à la rénovation est inimaginable. Ainsi le président de l'association en fait-il le constat lors d'une réunion:

"[...] L'association qui a mis l'écomusée en place, au moment où l'on a repris la chose, ils avaient dépensé pour l'achat de la maison, pour l'achat des collections; les installations, les aménagements, les vitrines etc., ils ont dépensé deux millions... et puis d'un seul coup pour une amélioration on passe à 15 millions, alors croyez-moi que dans la tête de Georges et puis dans la mienne... ils avaient travaillé eux pour mettre tout en place avec deux millions et puis d'un seul coup pour améliorer on en met $15 \ldots$ alors les chiffres ça nous paraissait quand même exorbitant, hein. Deux: nous on pense qu'un écomusée, ça s'aménage morceau par morceau...,

Nous reviendrons plus loin sur cette dernière affirmation: "un écomusée, ça s'aménage morceau par morceau" qui synthétise une divergence profonde de conception; retenons seulement ce refus de l'enveloppe financière qui rend compte d'un décalage de projection. Pour l'association, il s'agit d'améliorer, mais les références muséographiques demeurent celles de leurs expériences passées. Jusqu'à ce jour, aucune agence de muséographie professionnelle n'a été sollicitée dans l'écomusée. Les exigences de présentation ou de conservation préventive des bénévoles ne rencontrent pas celles des professionnels. Les associations considèrent généralement qu'il est possible de faire les choses en interne ou avec les artisans locaux, comme on le fait chez soi pour rénover sa maison. Un des éléments incompris des études de faisabilité concerne cette pratique courante de calculer selon des ratios au mètre carré. Ainsi le président rend-il compte de sa stupéfaction aux membres de son association:

"Dans l'aménagement d'espaces verts, il y a des sommes assez fabuleuses... Or, je crois que les amis de l'Écomusée on a déjà prouvé que l'on savait à peu près aménager, tondre, planter, etc. et que l'on pouvait faire... donc tout au long du projet, on pense que les chiffres qui ont été avancés ont été exorbitants, puisque ce Monsieur lle conservateun], il a fait un calcul savant: on construit X mètres carrés, pour la construction, 
ça doit être, je ne sais plus, je vous dis deux chiffres bidons, hein, ne les retenez pas, construction du mètre carré 10000 , muséographie pour remplir ces mètres carré 15000 , donc ça veut dire que, lui, il arrive à mettre 5000 francs le mètre carré, quand Georges ou... Raymond, etc. on travaille, bah nous, ca coûte... 100 balles le mètre carré, parce qu'on attend des opportunités..., on cbine quelque chose ici, on... etc., ça n'a rien à voir... c'est pour ça que les chiffres qui ont été avancés par ces... je répète le mot, ces technocrates, se sont des gens qui ont l'babitude de travailler avec de l'argent public, hein... je montrais l'autre jour le catalogue des fournitures aux Musées de France, hein, eh bien une malheureuse armoire en verre, c'est 8000 balles, y'a pas de problème... parce que l'on sait que c'est l'argent public qui va payer....

Outre l'argument populiste, aux relents poujadistes, qui accusent les fonctionnaires de dépenser sans compter l'argent public, et la révolte devant les prix du matériel professionnel eu égard aux dépenses connues jusque-là, on retiendra surtout l'écart souligné dans la façon de travailler. Aux logiques technocratiques de programmation, de financement et de chantier global s'oppose la méthode douce: celle qui consiste à attendre les opportunités. On voit bien s'opposer le caractère prométhéen du travail professionnel aux façons de faire de l'amateur qui a plus à voir avec le bricolage ou la passion du dimanche. Ainsi on cbine de-ci, de là. Or, loin d'être un inconvénient ou un élément négatif, ce travail à la petite semaine est pour les membres de l'association un critère positif de qualité. Non seulement parce que la méthode permet de faire des économies mais aussi parce qu'elle correspond à la façon dont "on veut vivre l'écomusée " un investissement par plaisir et comme lieu de socialisation. La débrouillardise suppose une organisation de travail qui repose sur l'économie informelle, les réseaux d'échange, les solidarités locales comme lieu du don. Parce qu'on se rend service, untel acceptera de venir réparer ou effectuer un service gratuitement, telle entreprise pensera à léguer quelques vieux matériels inutilisés, la femme d'un autre se proposera pour laver les costumes que l'on veut présenter. On se retrouvera ensemble à la prochaine fête organisée sur le site pour s'autocomplimenter. Dans cette atmosphère, les langues se délieront, les dons d'objets, hier "oubliés" au grenier, s'effectueront. D'autres projets seront évoqués.

Plus que tout, c'est la perte de ces moments, de cette façon de fonctionner qui est redoutée dans l'opération de rénovation. Les modes d'organisation informels, les débrouillardises locales, les arrangements de fortune, les trocs et échanges de services sont des caractères difficilement compatibles avec le fonctionnement administratif professionnel, celui "du monde des technocrates". Nous avons pu constater maintes fois qu'une grande partie du plaisir des bénévoles provenait du fát d'avoir agi sans moyens, "par débrouillardise". Ainsi tel service aurait dû être payé si on n'avait pas eu telle relation, telle connaissance, ou qu'untel ne disposait pas de certaines ressources. C'est ce savoir informel, cet échange non marchand qui donne une valeur au travail accompli. Surtout, it permet de montrer la supériorité du bénévole sur le professionnel qui malgré ses "grandes études" n'est pas maitre des réseaux de solidarité et des 
échanges locaux. Ce domaine échappe au pouroir et ne peut être qu'offert. 'Même si le résultat est peut-être moins abouti qu'il ne l'aurait été s'il avait fait l'objet d'une commande à quelqu'un du métier, que tel matériel récupéré est moins adapté que celui que lon aurait acheté ou fabriqué sur mesure, c'est surtout le principe d'économie communautaire qui préside. Or ce n'est pas seulement parce que le budget disponible est restreint et que l'on préfère faire avec ses petits moyens, sans recourir aux subventions. C'est surtout parce que cela correspond à un mode de fonctionnement rural qui exacerbe les solidarités, les services rendus. En cela, ce mode de gestion de l'écomusée fait perdurer des liens sociaux et des modes d'organisation du travail, eux-mêmes menacés par la modernité.

\section{LA GESTION DU STTE}

"Malgré tout, nous ne pratiquerons pas la politique de la chaise vide. Nous ne voulons pas mettre à l'assistance publique l'enfant que nous avons conçu et élevé jusqu'à 16 ans, puisque l'écomusée a 16 ans. C'est à l'adolescence, au moment où il grandit, où il cherche son orientation où il se développe que l'héritier a le plus besoin de ses parents, de ses amis, à moins qu'on ne veuille le sacrifier, le détruire, l'abandonner. Nous sommes déterminés à participer à l'élaboration et à la réalisation d'un projet qui réunit le maximum de XXX (babitants), afin que l'esprit des Écomusées soit sauvegardé, afin d'éviter le gaspillage des deniers publics et des deniers des contribuables. Et afin que cette réalisation demeure entre les mains des indigènes de la cité" (Allocution du président au Conseil municipal).

Le conflit est donc celui de la gestion: "on ne veut pas percire le bébê". Le problème est bien celui de la reconnaissance du bénévolat par les pouvoirs publics. Au-delà des discours généreux, volontiers tenus par les professionnels, de participation démocratique à la vie locale et d'appropriation de sa culture par l'implication des acteurs locaux, la réalité sur le terrain est moins éviclente. Quelle est la place des fondateurs du musée dans "l'éducation du bébé? "Ceci renvoie directement à la définition de l'écomusée. La population intervient-elle comme consommatrice des activités culturelles pensées pour elle? Comme partie prenante pour des opérations ciblées et délimitées? Comme actrice à part égale avec les professionnels? Comme acteur principal qui définit des opérations et pour lesquels des professionnels peuvent éventuellement intervenir en apportant leur compétence technique? Qui est au service de qui? En somme la question du pouvoir est posée.

Le local se trouve pris au piège d'accepter en bloc le projet de rénovation ou de le refuser et d'être accusé de laisser dépérir les collections. Car les professionnels sont soutenus par les partenaires financiers et la municipalité sait qu'elle ne pourra pas s'allier à un partenaire institutionnel au détriment des autres. Professionnaliser veut dire rationaliser l'organisation, définir des missions, des tâches, des objectifs. Surtout permettre le développement de réelles compétences pour accroître la qualité géné- 
rale du site. Ainsi le rapport au public est mis en avant,. le personnel d'accueil doit être formé et compétent. Surtout, la qualité de la prestation, en terme de contenu scientifique, doit être irréprochable. Impossible de transiger non plus sur les critères de présentation, de sécurité et de conservation des objets. Ceux-ci doivent être inventoriés, c'est-à-dire non seulement recensés mais documentés, classés, archivés selon dẹs critères répondant aux normes internationales. Il est impensable de rentrer dans l'inventaire constitutif des documents sous prétexte de faire plaisir à tel donateur, encore moins de rendre momentanément un objet pour dépanner un voisin, comme cela peut se faire couramment dans les petits musées! Les objets doivent être protégés, c'est-à-dire mis à distance. Autre forme de sacralisation que celle prônée par l'association: un écart est mis entre l'objet et la main qui peut lapprocher. Enfin, les animations et événements, même s'ils sont volontiers abandonnés à l'association d'amis de l'écomusée, ne sauraient admettre des prestations qui ne correspondent pas à l'image défendue en vue de se faire reconnaître comme institution sérieuse et crédible par ses pairs. Car les professionnels ont aussi pour motivation de disposer d'une institution de premier plan, dont la réputation régionale soit établie, dont l'originalité dans le milieu des musées soit affirmée au niveau national. En somme, l'association est nécessairement contrôlée, canalisée par les professionnels pour se conformer à leurs attentes. Or il est impossible à l'association de se penser écartée du pourvoir décisionnaire en matière de gestion et de limiter son rôle à une force de proposition et de mise en culvre des animations. Bref, l'association entend rester "maître à bord", c'est-à-clire actrice du lieu, et ne pas se réduire à jouer le rôle de figurants lors des fêtes locales. Revêtir le costume de vannier ou de brodeuse, participer à une séance de battage ou faire fonctionner une locomotive n'a pas le même sens quand il s'agit de céder aux clichés folkloriques d'un jour et quand c'est l'expression d'un sentiment collectif et d'un travail mené dans la durée.

\section{LES FORUES MUSÉOGRAPHIQUES RETENUES}

Mais l'argument de la gestion, s'il est plus conséquent que le problème des coûts nécessaires à l'opération de rénovation, n'est que l'arbre qui cache la forêt, car on pourrait théoriquement imaginer que le musée soit entièrement rénové, comme par un coup de baguette magique, qu'un écrin muséographique soit constitué, et que tout continue comme avant. Une fois leur mission civilisatrice effectuée - assainissement des locaur, mesures de conservation et de restauration des collections mises en place, meilleure fonctionnalité, réorganisation des espaces et des discours -, les professionnels et les institutionnels remettraient les clés à une association de gestion comblée et en expansion. En réalité, cela ne se passe jamais ainsi. La rénovation est toujours un instrument de dépossession d'une catégorie par une autre. Car c'est bien la question de la dépossession qui est posée. Derrière la gestion, se trouve la question du contenu du site: gérer peut-être mais gérer quoi? Imagine-t-on les professionnels 
ersonnel estation, isible de é et de lire non critères rer dans isir à tel - dépanis petits listance. in écart mations ociation ne corcomme tels ont $n$, dont ieu des ion est our se se penlimiter rations. ice du ocales. nce de quand expres-

e pro-

l'arbre musée qu'un avant. caux, es en unis sociapasse ession ossesntenu innels décider des espaces, de leur contenu, des discours et des présentations, selon leurs valeurs et références, et que l'association se retrouve ensuite chez elle? On n'habite jamais mieux les espaces que si on les a concus. En fait, c'est la conception même du musée, de l'esthétisation de ses formes qu'il faut interroger. Quelles sont les références, quels sont les types idéaux des uns et des autres et qu'est-ce qui les différencie? Bref, on n'échappe pas à la question du statut de l'objet, des façons de le mettre en scène, donc de la muséograpbie, du discours tenu, en somme de la médiation, et par conséquent des représentations sociales du musée et de ses fonctions. Et tout ceci renvoie plus largement à des cultures d'appartenance qui sont mises à jour dans les conflits opposant les parties. Pour comprendre les enjeux fondamentaux de la discorde, il faut donc s'intéresser aux conceptions auxquelles se réfèrent les uns et les autres sur les missions dévolues à un écomusée, aux représentations sociales du public que l'on désire accueillir, de ce que l'on imagine de ses attentes et ce que l'on désire lui transmettre. Pour cela, il faut analyser les orientations muséologiques et les formes muséographiques que chacun entend mettre en cuvre. Nous ne pouvons, dans le cadre de cet article, aborder toutes ces questions pourtant complémentaires. Mentionnons seulement l'idéal muséal du bénévole.

\section{LES FIGURES DU MUSÉE IDÉAL}

Contrairement à toute attente, le bénévole ne veut pas que son musée tende à ressembler au Louvre ou à la Cité des Sciences et de l'Industrie, auquel il n'identifie pas sa culture, alors que tous les efforts du professionnel visent à un idéal. Même si les grandes institutions prestigieuses sont critiquées pour tel ou tel aspect, il existe dans le fond un paradigme commun sur ce que doit être un musée moderne. Sans s'appesantir sur les services, qui font du musée un nouvel espace public (Rasse, 1999), et que le bénévole est en mesure d'admettre, c'est le cour même du dispositif qui fait distinction. Aux salles de plus en plus light des professionnels, que ce soit en terme d'artefacts ou de discours, à la prédominance de la forme et du design, des matériaux nobles et clinquants des salles des musées rénovés, le bénévole préfère les salles surchargées, manifestant la richesse de leur accumulation, les collections denses et pléthoriques. On aurait du reste tort de se moquer, les amateurs de musée d'art ont souvent manifesté, dans les débuts de cette nouvelle mode muséographique, les plus grandes réticences avant d'abdiquer devant le corps des conservateurs. Aujourd'hui, le public reprend le plus souvent à son compte cet idéal de clarté dans l'énonciation, l'épuration des contenus, mais ceci n'a pas toujours été le cas. Les salles d'exposition dépourvues de collections ont d'abord fát figure de bizarrerie. C'est à cette première image que les bénévoles se réfèrent. Ils conçoivent un musée où l'abondance signifie la richesse des collections, et devient surtout synonyme de la valeur et de l'importance de la culture locale. Bref, limiter les présentations porte quelque part atteinte 
à l'intégrité et à la grandeur du patrimoine. Cette atteinte à l'identité est d'autant plus insupportable quand elle est mise en cuvre par des "étrangers" au territoire et à sa culture. Forme de castration symbolique, le remaniement et surtout la restriction des objets présentés, pour les consigner en réserve - c'est-à-dire relégués hor's du regard, dans l'ombre et le secret -, sont source de polémique. Les professionnels ont tendance à minimiser le problème, voire à le dissiper derrière des jeux de langage et d'écriture. Toutefois, les bénévoles sentent confusément que se joue là une divergence importante, et le manifestent en se montrant critiques vis-à-vis de l'utilité d'une réserve et de médiations dans le futur musée.

Ainsi le professionnel envisage des médiations, des mises en scènes, des critères de sélection des expôts, dans lesquels ne se reconnaissent pas les bénévoles. Par exemple, ceux-ci ne veulent pas, à tort ou à raison, des audiovisuels pour leur expliquer leur territoire. C'est étonnant, mais le président avertit: "il ne faut pas détourner l'attention des visiteurs avec dés textes ou des vidéos... ". Ce qui est idéalisé par les professionnels des musées fait figure ici de critique. Inutile de recourir à de gros moyens muséographiques, à des recherches savantes pour élaborer des textes inscrits sur des supports toujours plus design: l'idéal de musée est autre. Or, qu'est-ce qui nous certifie que ce bénévole ait tort? Après tout, ce n'est peut-être pas un hasard si les classes populaires vont davantage dans les musées qui ne sont guère ou tout juste reconnus comme tels par la Direction des Musées de France... Les études de publics montrent que les classes socialement moins favorisées se pressent davantage dans les musées techniques, les musées de collectionneur, les petits musées de "terroir". Les musées qui présentent des séries d'objets, comme les musées de l'automobile (à Sochaux, Mulhouse ou La Clayette), les différents musées de la maquette, ou encore le musée Grévin, sont également davantage plébiscités par les classes populaires que les musées aux concepts et aux discours élaborés. On peut même postuler que les musées techniques qui font l'objet de rénovations, qui passent d'un lieu d'accumulation à un lieu de discours sur le rapport des hommes à ces objets, dans toutes leurs dimensions, voient leur public se modifier. Il ne s'agit pas de chercher des explications autres que sociologiques au phénomène. Simplement, doit-on s'étonner que les classes intellectuelles s'identifient davantage à des lieux de discours, et les classes dominées à une culture plus matérielle et plus tangible? On pourrait aisément relier cette conception idéale du musée à un habitus de classe et aux valeurs qui y sont associées. C'est aussi que le musée, évocateur des objets relégués d'un passé révolu, doit conserver un aspect de vaste réserve et ne pas ressembler à un livré41. L'accumulation est signe de richesse, preuve d'un héritage et d'un dépassement, d'une eschatologie. Il ne s'agit pas de construire une réflexion savante sur le passé et sur ses liens au présent mais d'être saisis par l'impact d'une densité qui impose une posture.

voles st entende avec le: faite $v e$ entendk de cô̂l rénoval effectiv élémen même lérable progras faire di inport. tectes tion d' pour d et avo: visions tions, de pré tique, image

C

ou écr forme de viv: de la F tion $\mathrm{d}$ l'écorr dégag.

l'écorr bénév celui de l'é vision passé leur $c$ même comn les se davar sionn gênax quatr 
identité est -e par des ymbolique, s, pour les zard, dans onnels ont :e des jeux infusément an se monms clans le

en scènes, connaissent ou à raison, mant, mais iteurs avec fessionnels à de gros laborer des de musée tort? Après laires vont 2 reconnus études de es se prescollectiont des séries ulhouse ou e le musée populaires peut même rations, qui - le rapport voient leur ions autres tonner que le discours, is tangible? :à un habiussi que le it conserver L'accumulapassement, ion savante sar l'impact
DES VISIONS DU MONDE HÉTÉROGÈNES

La problématique est celle du rapport du local au global. Les bénévoles se sentent porteurs et investis d'un projet, d'une identité locale. Ils entendent réaliser le musée qui les représente, localement, c'est-à-dire avec les forces disponibles sur place. Cela signifie qu'ils n'entendent pas faire venir des Parisiens, ni même des gens de la ville avoisinante. II s entendent réaliser la rénovation à l'échelle de leur communauté, en terme de coût, d'échelle de fréquentation espérée, mais aussi d'envergure de rénovation. "On veut refaire petit à petit, un endroit après l'autre". C'est effectivement la démarche suivie jusque-là: chaque année on ajoute un élément, pour construire un amalgame, une sorte d'empilement, avec la même logique qui préside à l'accumulation des vitrines et qui paraît intolérable aux professionnels investis dans une logique technocratique de programmation. L'argument du professionnel est à l'opposé, il s'agit de faire décoller radicalement la fréquentation, de trouver des financements importants, de faire travailler des gens de renoms, notamment les architectes (mais aussi des graphistes "branchés"...), et de penser la rénovation d'un point de vue global, c'est-à-dire de tout refaire en même temps pour donner une signification forte. Il faut programmer sur le long terme et avoir une cohérence générale de discours. S'affrontent bien ici deux visions du monde, si ce n'est deux formes de pensée, avec des conceptions, des représentations de l'espace, de l'ordonnancement des espaces, de présentation des objets, et sans doute de choix des objets, d'esthétique, voire d'éthique muséographique, qui rend compte au-delà d'une image de présentation de soi.

C'est la définition même de l'institution qui est ainsi en cause: musée ou écomusée? Les bénévoles accusent les professionnels de vouloir transformer leur écomusée en musée, c'est-à-dire selon eux "de quelque chose de vivant en quelque chose de mort". On peut repérer une double inversion de la problématique telle que la posait Duncan F. Cameron entre la conception du musée temple et du musée forum et la compléter des théories de l'écomuséologie (Cameron, 1971, p. 77). Cette nouvelle lecture permet de dégager de nouveaux types d'orientations muséologiques. Dans le cas de l'écomusée étudié, c'est la représentation traditionnelle du musée que les bénévoles veulent mettre en œuvre d'un point de vie muséographique, celui de la série d'objets, de la collection. Conception qui s'oppose à la celle de l'écomusée où la collection doit être au service de l'Homme et d'une vision globale, d'une dynamique, bref où l'objet n'est plus tourné vers le passé mais vers le présent. À ce titre ce sont les professionnels, qui, dans leur orientation, sont les plus proches de la mission de l'écomusée. En même temps, les bénévoles veulent être les acteurs du projet, tels que le commande la définition de l'écomusée. Ils ne veulent pas d'un lieu géré par les seuls techniciens comme dans le nusée traditionnel. Ils sont à ce niveau davantage en adéquation avec la définition de l'écomusée que les professionnels portés à occulter une association considérée sourvent comme gênante. On peut par conséquent ajouter deux nourveaux modèles. Ces quatre modèles organisent les musées aujourd'hui. 
1. Le musée traditionnel: tourné vers le passé, il présente des objets pour eux-mêmes. Les objets sont sacralisés. Le musée est géré par des professionnels. C'est l'archétype du musée de beaux-arts, contre lequel s'est élevée la nouvelle muséologie et tous les courants critiques du musée.

2. L'écomusée: l'objet est prétexte à discours. Le passé sert à appréhender le présent, des liens sont proposés. La collection est au service d'une vision globale et dynamique de l'Homme. L'établissement est un lieu de vie permettant de renforcer des liens sociaux. Il est l'expression d'une communauté et nécessite une implication des acteurs locaux. Cette forme d'expérience muséale ne semble survivre qu'en de rares endroits (écomusée du Fier Monde à Montréal). Le plus souvent, l'écomusée conserve les apparences d'une participation locale que l'analyse approfondie dément rapidement. La professionnalisation a conduit à faire disparaitre la population locale et, par là même, la vocation de l'écomusée comme lieu d'expression démocratique. En ce sens, le terme même d'écomusée, dans son sens originel, est le plus souvent discutable.

3. Le musée professionnel des années quatre-vingt-dix. Héritier de la vision critique sur l'objet, sur le musée et la collection, il entend contextualiser et se servir du musée pour développer un discours antbropologique. C'est la conception contemporaine du "musée de société" développée par les théoriciens de la muséologie. Il affirme la nécessité de la professionnalisation, que ce soit au niveau de la gestion ou de la conception, amenant une certaine uniformisation des contenants, si ce n'est des contenus. Les professionnels sont des spécialistes qui développent des discours sur les acteurs d'un territoire. C'est une vision quelque peu technocratique de la culture, puisque la parole est canalisée par le professionnel, même si elle peut être confiée à l'occasion à des acteurs pour des moments privilégiés (exposition sur telle communauté). Toutefois, la délégation de la parole demeure largement sous contrôle. Les illustrations les plus probantes en sont données par le musée de la Civilisation, le Musée dauphinois, l'écomusée de Fresnes..

4. L'ethno- ou socio-résistance des intéressés, "des indigènes". Ceux-ci entendent continuer à concevoir, présenter et gérer leur territoire et leurs sujets d'intérêt comme ils l'entendent. Cependant, ils organisent le lieu selon leurs représentations sociales du musée et donc de la culture cultivée (ce que doit être un beau musée), risquant de mettre en œuvre des perspectives rétrogrades de l'identité et des techniques muséographiques jugées dépassées par le professionnel (diorama, etc.). Ils sont nécessairement en retard sur les modes de la muséographie, d'autant que celles-ci deviennent financièrement inaccessibles du fait des nouvelles technologies.

On voit donc s'opposer, au travers de la muséographie, par la médiation même, une vision identitaire locale à une vision globalitaire prônant un modèle de référence universaliste. Les critères d'une rénovation de musée, de ce qu'il doit être, de ce qu'il doit montrer et comment, selon quelle lecture pour le visiteur étranger au local, correspondent à des normes standardisées pour le professionnel. La médiation comporte ainsi des enjeux politiques évidents. La différence de traitement de la cul- ture locale pc de qui les tran versel (d'un : muséographic professionnel. munauté et $n$ deux cas, il s' tures de légiti liens socianux mêmes. À mo unit véritables Les pron le projet de l'. tion en parall entre les partj dossier de la locaux, n'aya les réalisatior dans les salle: faire opérer l dents un mus ser un vrai m naissent pas logique de I considérée qui ne possèr sans en com: la population des experts. 1 rés par les $\mathrm{F}$ scientifiquem dables du po est certain qu lls sont auss contradiction que soit le demeure disc on pourra rét sente qu'une et ne regrou: idéologie su: Mais il deme que, selon $1 \epsilon$ acteurs qui $\mathrm{c}$ lectif exprim au service de ment correc contredire et 
les objets par des re lequel iques du

à appréI service it est un cpression locaux. de rares l'éconull'analyse lit à faire l'écomule même

tier de la 1 contexbropolođévelopté de la concepn'est des sent des reu techfessionour des efois, la strations ation, le

Ceurx-ci et leurs eu selon ivée (ce pectives 5 dépasn retard it finan-

par la jalitaire rénovaminent ndent à mporte a la cul- ture locale pose le problème des messages transmis, mais est inséparable de qui les transmet, de qui parle? Le professionnel parle au nom de l'universel (d'un discours de vérité établi scientifiquement et de techniques muséographiques à la mode) et donc de sa culture d'appartenance de professionnel. Le bénévole, lui, prétend parler comme acteur d'une communauté et met en scène une autre mythologie identitaire. Car dans les deux cas, il s'agit de reconstmuction du monde, mais en fonction de postures de légitimations différentes. Dans les deux cas, on a construction de liens socianx autour de projets de rénovation, mais il ne s'agit pas des mêmes. A moins que le lien social le plus opérant ne soit le conflit qui unit véritablement les partisans en les opposant!

Les pronostics les plus probables vers lesquels semble s'acheminer le projet de l'écomusée étudié dans le cadre de cet article sont une évolution en parallèle des deux conceptions avec une simulation de dialogue entre les parties. Ainsi, les architectes et les professionnels font avancer le dossier de la rénovation d'un point de vue technique et financier. Les locaux, n'ayant pas les mêmes références, n'imaginent pas concrètement les réalisations. À l'heure des travaux et de l'installation de l'exposition dans les salles, il sera trop tard pour que l'association puisse s'opposer et faire opérer un retour un arrière. Les professionnels fourniront aux résidents un musée selon leur standard et leur référence. Ils auront pu réaliser un vrai musée professionnel, même si les "autochtones" ne s'y reconnaissent pas et désertent l'endroit enfin investi par les touristes. Cette logique de la compétence du spécialiste, imposée à une population considérée comme ignorante, est symptomatique d'un fonctionnement qui ne possède de démocratique que le nom. Aux élus ayant fait un choix sans en comprendre visiblement les enjeux, aux souhaits d'une partie de la population locale investie dans le projet, s'imposent pour finir les choix des experts. Cette remarque ne juge en rien de la validité des choix opérés par les professionnels sur le fond, phus responsables, plus valides scientifiquement, plus cohérents sur le plan muséologique, plus défendables du point de vue idéologique et politique. Sur le plan intellectuel, il est certain que les choix des professionnels sont de loin plus stimulants. Ils sont aussi plus responsables, car nous n'avons pu détailler ici les contradictions nombreuses et les voltes-faces des bénévoles. Mais, quel que soit le bien-fondé des solutions prescrites par le spécialiste, il demeure discutable que celui-ci puisse les imposer aux intéressés. Certes, on pourra rétorquer que, d'une façon générale, une association ne représente qu'une infime part de la population, qu'elle n'est pas représentative et ne regroupe finalement que des opposants instrumentalisés par une idéologie suspecte, réactionnaire, passéiste et rétrograde. Cela est vrai. Mais il demeure que ce sont les personnes mobilisées dans l'opération et que, selon le strict point de vue de la théorie écomuséale, ce sont les acteurs qui comptent, puisqu'ils expriment finalement le seul souhait collectif exprimé par les habitants du territoire. Le professionnel doit-il être au service de l'expression de la population quand celle-ci est politiquement correcte, l'instrumentaliser et la superviser, s'en émanciper, la contredire et lui imposer d'autres logiques quand elle ne correspond plus 
à ses souhaits? Sont posées les limites aux théories de l'écomuséologie. Il serait bon d'en prendre acte et de redéfinir le terme d'écomusée. Faut-il ne pas suivre l'avis de la population quand cet avis n'est plus du goût du spécialiste? On voit bien où peuvent conduire les aléas du populisme mais aussi, à l'extrême, les suffisances d'une élite persuadée de détenir les lumières de la raison. Cette ambivalence s'exprime à une petite échelle au niveau des choix faits pour une rénovation de musée, mais ce sont les mêmes logiques qui président sur des dossiers politiquement ou scientifiquement plus sensibles.

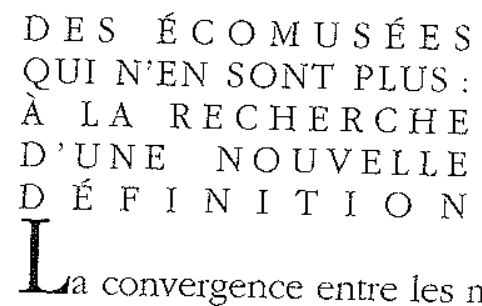
les écomusées, pour les modes de gestion et de fonctionnement, les techniques muséographiques et de conservation, conduit à une normalisation et à une standardisation. À tel point qu'on ne voit plus très bien la spécificité des écomusées aujourd'hui. Peut-être la demande sociale de prise de parole publique n'est-elle plus tout à fait d'actualité? Le témoignage par l'objet l'affirmation d'une identité et le recours au patrimoine comme lieu de ressource pour conduire une politique locale ne passe-t-il pas par d'autres voies? Après tout, l'expérience écomuséale est contemporaine de crises culturelles, sociales, économiques, industrielles, de changement dans les moeurs et les modes de vie, d'urbanisation et de transition entre deux mondes, celui du local au village planétaire, si ce n'est à la mondialisation. Peut-être cette crise est-elle digérée: les sites doivent alors devenir des musées classiques, ou encore trouver d'autres fonctions, notamment touristiques (avec la génération des scopes et autres parcs), de confirmation culturelle et mythologique (avec des sites légendes), économiques (avec les économusées et leur lieu de vente), de communication (comme les musées d'entreprise)... Le développement des nouvelles technologies de l'information et de la communication, notamment de l'Internet, fait peut-être se déplacer le débat et l'investissement démocratique sur d'autres terrains. Liddentité se vit ailleurs et autrement, à moins que la virtualité ne rende caduque toute volonté de conscience citoyenne et politique. Une politique locale fondée sur la mémoire intéresse t-elle encore à l'heure de l'invention de soi? L'identité, après avoir été relativisée, est-elle condamnée au seul fantasme? Sauf à inventer d'autres missions et à redéfinir l'écomusée autrement, un grand nombre d'acteurs semble d'accord pour ranger sagement les théories de l'écomuséologie au musée.. Centre de recherche sur le culture et les musées (CRCM) 
Éologie. Il

śe. Faut-ii

1 goût du

ropulisme

e détenir

ne petite

- mais ce

zment ou

snnels et

les tech-

alisation

sécificité

le parole

$r$ l'objet,

1 de res-

d'autres

ises cul-

lans les

re deux

lisation.

enir des

t touris-

n cultu-

les éco-

musées

nforma-

-être se

errains.

$\because$ rende

olitique

vention

sul fan-

ement,

ss théo-

S. C.
NOTES

1. Cet article reprend une partie de l'analyse proposée dans un livre à parâttre sur la question.

2. Les citations d'Hugries de Varine (HdV) sont extraites des différents articles de l'auteur, repris dans les volumes 1 et 2 de Vagues (1992 et 1994).

3. Patrick Le Nouenne fait remarquer que la soi-disant expression du local est souvent représentative d'une petite partie de la population qui instrumentalise l'écomusée, "Un écomusée ce r'est pas un musée comme les autres " (1978).

4. C'était également, selon André Desvallées, une caractéristique de la fréquentation des deux galeries du Musée National des Arts et Traditions Populaires lorsqu'elles étaient ouvertes simultanément au public: la Galerie culturelle et la Galerie d'études.

\section{RÉFÉRENCES BIBLIOGRAPHIQUES}

Augé (M.) (éd.). 1992. Territoires de la mémoire. Thonon-les-Bains: Éd. cle LAalbaron, 1992.

Barbe (J.-M.). 1985. "Présence et avenir du passé. Contribution à une problématique des nouvelles muséologies. Les Cabiers de l'Animation, 51.

Cameron (D. F.). 1971. "Le Musée: temple ou forum. Repris in Vagues: Une anthologie de la nouvelle muséologie, vol. 1. Textes choisis et présentés par André Desvallées. Mâcon / Savigny-LeTemple: W/ MNES.

Cotton (J.-P.). 1998. "L'Identitaire, notion conflictuelle et ambiguë", L'ARA, 43, p. 32-33.

Desvallées (A.). 1992. "Présentation", in Vagues: Une anthologie de la nouvelle muséologie, vol. 1. Textes choisis et présentés par André Desvallées. Mâcon / Savigny-Le-Temple: W / MNES.

Duclos (J-C.), Veillard (J.-Y.). 1992. "Musée d'ethnographie et politique". Museum, 175, p. 129-132.

Finkielkraut (A.). 1987. La Défaite de la pensée. NRF.

Hubert (F.). 1985. "Les écomusées en France: contradictions et déviations. Museum, 148, p. 186.

La Muséologie selon Georges Henri Rivière. 1989. Paris: Dunod.

Le Nouenne (P.). 1978. "Un écomusée ce n'est pas un musée comme les autres. Repris in Vagues: une anthologie de la nouvelle muséologie, vol. 1. Textes choisis et présentés par André Desvallées. Mâcon / Savigny-LeTemple: W/ MNES.

Mairot (P.). 1992. "L'Objet de l'écomuséen, p. 23-35 in Temitoires de la mémoire / sous la direction. de Marc Augé. Thonon-les-Bains: Éd. de L'Albaron.

Mairot (P.). 1997. "Musées et société". Ethnologie francaise, $\mathrm{Vol} 27(3)$, p. 34低356.

Prado (P.). 1995. "L'Ethnologie française au musée? Ou un nouveau musée de l'ethnologie de la France? ". Terrain, 25 , sept., p. 147-157. 
Prasch (H.). 1992. "Musées régionaux, musées locaux: quel dialogue? ". Museum, 175, 44(3).

Rásse (P.). 1999. Les Musées à la lumière de l'espace public. Paris: Éd. I'Harmattan.

Rivière (Georges Henri). 1992 (19711985). "L'écomusée, un modèle évolutif", p. 440-445 in Vagues: Une anthologie de la nouvelle muséologie, vol. 1. Textes choisis et présentés par André Desvallées. Mâcon / Savigny-LeTemple: W / MNES.

Varine ( $\mathrm{H}$. de). 1969. "Le musée au service de lhomme et du développement". Repris p. 49-68 in Vagues: Une anthologie de la nouvelle muséologie, vol. 1 / textes choisis et présentés par André Desvallées. Mâcon / Savigny-LeTemple: W / MNES, 1992.

Varine (H. de). 1978. "L'Écomusée" La Gazette (Association des musées canadiens), 11, p. 28-40. Repris p. 446-487 in Vigues: Une anthologie de la nouvelle muséologie, vol. 1 / textes choisis et présentés par André Desvallées. Mâcon / Savigny-Le-Temple: W / MNES, 1992.

Varine (H. de). 1979. "Le musée peut tuer ou... faire vivre". Techniques et Arcbitecture, 326, sept. Repris p. 65-73 in Vagues: Une anthologie de la nouvelle muséologie, vol. 2 / textes choisis par Marie-Odile de Bary, André Desvallées, Françoise Wasserman et présentés par André. Desvallées. Mâcon / Savigny-Le-Temple: W / MNES, 1994.

Varine (H. de). 1982. "Viol et vol des cultures: un aspect de la dégradation des termes de l'échange culturel entre les nations", p. 152-157 in Collections passion, Neuchâtel: Musée d'ethnographie. Repris p. 92-110 in Vagues: Une anthologie de la nouvelte muséologie, vol. 2 / textes choisis par Marie-Odile de Bary, André Desvallées, Françoise Wasserman et présentés par André Desvallées. Mâcon / Savigny-LeTemple: W / MNES, 1994 


\section{RÉSUMÉS}

\section{À}

partir des données recueillies lors d'une enquête sur le terrain auprès d'une association d'amis d'un écomusée, l'article vise à étudier les conceptions et les représentations sociales de bénévoles confrontés à des professionnels intervenant également sur le site. Les conceptions, tant du contenu des expositions, de la scénographie, des médiations, des projets culturels et scientifiques que des publics à accueillir, révèlent des projets hétérogènes. Il s'agit d'appréhender des logiques sociales particulières puis de les recontextualiser dans une approche plus globale. En s'appuyant sur une relecture des théories fondatrices de l'écomuséologie, il est possible d'examiner l'évolution des conceptions en la matière et plus généralement des tendances muséologiques en cours.

\section{$A$ study of an Ecomuseum Association bas been} conducted in order to differentiate between volunteers and Museum professionals' interpretations. Their respective approaches to the contents of the exbibitions, to their stageing, to their presentation and to the cultural and scientific projects as well as to the audiences they aim at attracting reveal great beterogeneousness. The goal of this paper is to a study specific social approaches, to recontextualize them and to present them in a more general perspective. Starting from the founding theories of the Ecomuseum, it si possible to show the evolution in this particular field as well as the general trends in present day museology.

\section{A}

partir de los resultados de una investigación sobre una asociación de amigos de un "ecomuseo", el artículo propone estudiar las concepciones y representaciones sociales de voluntarios confrontados a profesionales que intervienen también en éste. Las concepciones, tanto del contenido de las exposiciones, de la escenográfia, de las mediaciones, de los proyectos culturales y cientificos como de] público que acoger, revelan proyectos heterogéneos. Se trata de examinar lógicas sociales específicas y luego de enforcarlas dentro de un marco más global. Basándose en una nueva lectura de las teorías fundadoras de los "ecomuseos", es posible examinar la evolución de dichas concepciones y, más ampliamente, de las tendencias museológicas actuales. 